University of Nebraska - Lincoln

DigitalCommons@University of Nebraska - Lincoln

April 2001

\title{
Solution Structure of $B$. subtilis Acyl Carrier Protein
}

\author{
Guang-Yi Xu \\ Department of Biological Chemistry, Wyeth Research, Cambridge, MA 02140, USA
}

Amy Tam

Department of Biological Chemistry, Wyeth Research, Cambridge, MA 02140, USA

Laura Lin

Department of Biological Chemistry, Wyeth Research, Cambridge, MA 02140, USA

Jeffrey Hixon

Millennium Pharmaceuticals, Inc., Cambridge, MA

Christian C. Fritz

Millennium Pharmaceuticals, Inc., Cambridge, MA

See next page for additional authors

Follow this and additional works at: https://digitalcommons.unl.edu/chemistrypowers

Part of the Chemistry Commons

Xu, Guang-Yi; Tam, Amy; Lin, Laura; Hixon, Jeffrey; Fritz, Christian C.; and Powers, Robert, "Solution Structure of B. subtilis Acyl Carrier Protein" (2001). Robert Powers Publications. 8.

https://digitalcommons.unl.edu/chemistrypowers/8

This Article is brought to you for free and open access by the Published Research - Department of Chemistry at DigitalCommons@University of Nebraska - Lincoln. It has been accepted for inclusion in Robert Powers Publications by an authorized administrator of DigitalCommons@University of Nebraska - Lincoln. 


\section{Authors}

Guang-Yi Xu, Amy Tam, Laura Lin, Jeffrey Hixon, Christian C. Fritz, and Robert Powers 


\section{Solution Structure of B. subtilis Acyl Carrier Protein}

\author{
Guang-Yi Xu*, Amy Tam*, Laura Lin*, \\ Jeffrey Hixon ${ }^{\dagger}$, Christian C. Fritz ${ }^{\dagger}$, and \\ Robert Powers*
* Department of Biological Chemistry, Wyeth Research, Cam- bridge, MA 02140, USA
† Millennium Pharmaceuticals, Inc., Cambridge, MA 02140, USA
$\ddagger$ Corresponding author: Robert Powers

\section{Summary}

Background: Acyl carrier protein (ACP) is a fundamental component of fatty acid biosynthesis in which the fatty acid chain is elongated by the fatty acid synthetase system while attached to the 4'-phosphopantetheine prosthetic group (4'$\mathrm{PP}$ ) of ACP. Activation of ACP is mediated by holo-acyl carrier protein synthase (ACPS) when ACPS transfers the 4'-PP moiety from coenzyme A (CoA) to Ser36 of apo-ACP. Both ACP and ACPS have been identified as essential for $E$. coli viability and potential targets for development of antibiotics.

Results: The solution structure of $B$. subtilis ACP (9 kDa) has been determined using two-dimensional and three-dimensional heteronuclear NMR spectroscopy. A total of 22 structures were calculated by means of hybrid distance geometrysimulated annealing using a total of 1050 experimental NMR restraints. The atomic rmsd about the mean coordinate positions for the 22 structures is $0.45 \pm 0.08 \AA$ for the backbone atoms and $0.93 \pm 0.07 \AA$ for all atoms. The overall ACP structure consists of a four $\alpha$-helical bundle in which 4'-PP is attached to the conserved Ser36 that is located in a helix II.

Conclusions: Structural data were collected for both the apo and holo forms of ACP that suggest that the two forms of ACP are essentially identical. Comparison of the published structures for E. coli ACP and actinorhodin polyketide synthase acyl carrier protein (act apo-ACP) from Streptomyces coelicolor A3(2) with $B$. subtilis ACP indicates similar secondary structure elements but an extremely large rmsd between the three ACP structures $(>4.3 \AA)$. The structural difference between $B$. subtilis ACP and both $E$. coli and act apo-ACP is not attributed to an inherent difference in the proteins, but is probably a result of a limitation in the methodology available for the analysis for E. coli and act apo-ACP. Comparison of the structure of free ACP with the bound form of ACP in the ACP-ACPS complex reveals a displacement of helix II in the vicinity of Ser36. The induced perturbation of ACP by ACPS positions Ser36 proximal to coenzyme $A$ and aligns the dipole of helix II to initiate transfer of 4'-PP to ACP.

Keywords: ACP, ACPS, fatty acids biosynthesis, NMR, solution structure

\section{Introduction}

The biosynthesis of fatty acids consists of a series of reactions that are catalyzed by specific enzymes [1]. The organization of the enzymatic activity is significantly different between eukaryotic cells and prokaryotic and plant cells. In eukaryotic cells, large multifunctional enzymes exist with distinct domains that are associated with a particular function. Conversely, in prokaryotic and plant cells, the various enzymatic activities are associated with individual proteins that are loosely associated with each other. Acyl carrier protein $(\mathrm{ACP})$ is a discrete, small acidic protein $(9 \mathrm{kDa})$ in prokaryotic and plant cells that plays an essential role in fatty acid biosynthesis, whereas, in animal tissue, ACP is a subunit of the fatty acid synthetase (FAS). ACP is the carrier of fatty acids during fatty acid biosynthesis and is responsible for acyl group activation [1, 2 and 3].

A unique feature of ACP is the presence of the 4'phosphopantetheine (4'-PP) prosthetic group. The 4'-PP moiety is attached through a phosphodiester linkage to a specific conserved serine residue found in all ACPs. ACP exists in both an active (holo) and inactive (apo) form in which activation of ACP is mediated by holo-acyl carrier protein synthase (ACPS). ACPS transfers the 4'-PP moiety from coenzyme A to Ser36 of apo-ACP to produce holo-ACP and 3', 5 '-ADP in a $\mathrm{Mg}^{+2}$-dependent reaction. During biosynthesis of a long-chain fatty acid, the fatty acid chain is attached to ACP via a thioester linkage to the terminal cysteamine thiol of the 4'-PP prosthetic group, where it is then elongated by the FAS system. A potential function of the 4'-PP prosthetic group is to act as a tether that serves to transfer the growing fatty acid chains between the various enzymes or active sites in the FAS system [4].

ACP is a central component of and plays a fundamental role in fatty acid and other biosynthetic pathways that require acyl transfer steps [5, 6, 7, 8 and 9]. The activation of ACP by ACPS is critical to this function in which both ACP and ACPS were identified as being essential for the viability of E. coli [10 and 11]. Furthermore, both ACP and ACPS are viable targets for a drug discovery program, since the enzymes are essentially unique to prokaryotic cells. This is made further evident by previous efforts to determine the structures for $E$. coli ACP and act apo-ACP prior to the structure determination of $B$. subtilis ACP reported herein $[12,13,14$ and 15]. Since the activation of ACP is mediated by its interaction with ACPS, interfering with either the activity of ACPS or the binding interaction of ACPS with ACP may prove to be a valuable approach for developing novel antibiotics. Toward this goal, we present the NMR resonance assignments and the determination of the solution conformation of $B$. subtilis ACP. 
(a)

(c)
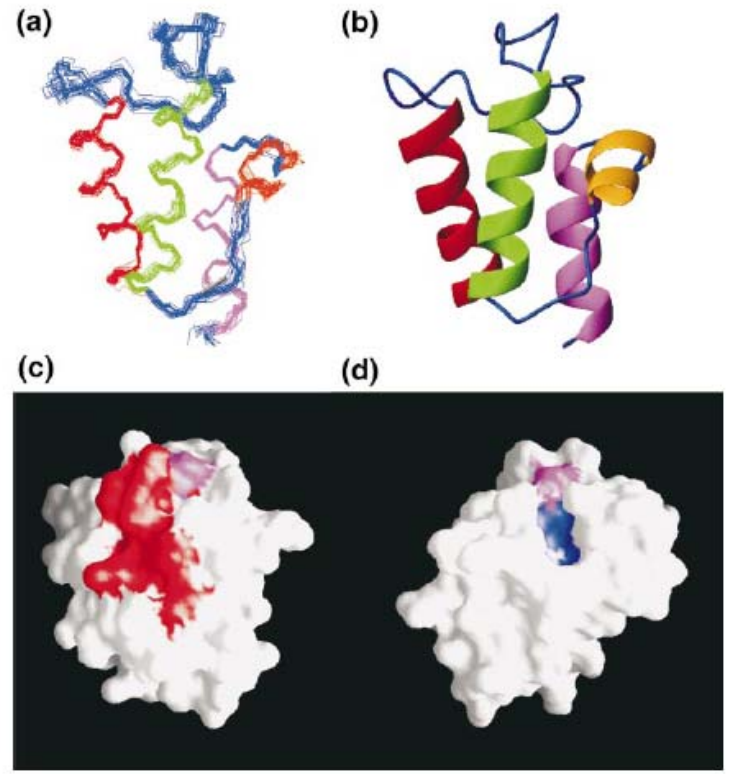

Figure 1. Solution Structure of $B$. subtilis ACP

(a). The best-fit superposition of the $\mathrm{Ca}$ traces of the 22 members of the final ensemble of NMR-derived structures of $B$. subtilis ACP.

(b) Ribbon diagram of the averaged and minimized NMR structure of $B$. subtilis ACP. For (a) and (b), helixes I-VI are colored red, green, orange, and magenta, respectively. The crossover loops are colored blue.

(c) Residues that exhibit two distinct sets of ${ }^{15} \mathrm{~N} / \mathrm{NH}$ chemical shifts associated with the apo and holo forms of $B$. subtilis ACP are colored red, and S36 is colored magenta.

(d) The hydrophobic pocket composed of residues F28, V39, V40, L42, V43, L46, and V65 (blue) is illustrated. The surface corresponding to S36, which is proximal to the hydrophobic pocket, is colored magenta

\section{Results and Discussion}

\section{Secondary Structure Analysis and Structure Determination}

The regular secondary structure elements of ACP were identified through a qualitative analysis of sequential and medium- range NOEs obtained from the ${ }^{15} \mathrm{~N} /{ }^{13} \mathrm{C}$-edited NOESY spectra, $\mathrm{NH}$ exchange rates, $\varphi / \psi$ dihedral torsion angles from Talos [16], and the ${ }^{13} \mathrm{C} \alpha$ and ${ }^{13} \mathrm{C} \beta$ secondary chemical shifts [17]. The secondary structure of $B$. subtilis ACP is composed of four helical regions that correspond to residues A1-R14 (al), S36-F50 (all), D56-E60 (allI), and G66-Q75 (aVI). Three loop regions of various lengths connect the four helices.

The calculated three-dimensional structure of $B$. subtilis ACP is well defined by 1050 distance restraints, 54 hydrogen bond restraints, and 92 torsion angle restraints. The $\alpha-$ helical regions of the calculated structure are similar to the predicted secondary structure elements. Figure 1a shows the best-fit superposition of the $\mathrm{Ca}$ traces for the final ensemble of 22 structures, illustrating the consistency within the a helix "framework" and variability of one long (20 residues) and two short interhelix loops (3 and 6 residues). The ribbon diagram of the ensemble average-minimized structure is shown in Figure 1b. The NMR structure is well de- fined, as evident by the atomic rmsd of the 22 simulated annealing structures about the mean coordinate positions where the backbone and all atoms' rmsd are $0.45 \pm 0.08 \AA$ and $0.93 \pm 0.07 \AA$, respectively. For residues that are only in secondary structure regions, the backbone and all atoms' rmsd are $0.35 \pm 0.08 \AA$ and $0.85 \pm 0.06 \AA$, respectively. The $B$. subtilis ACP NMR structure is consistent with a high-quality structure based on PROCHECK and Ramachandran analysis [18 and 19]. A Ramachandran plot of the average-minimized structure shows a total of $83.1 \%$ of the residues in the most favored region, $12.7 \%$ in the additional allowed region, and $2.8 \%$ in the generously allowed regions, with only 1 residue (Val17) in a disallowed region. Val17 is a member of the twist turn, which is located in the long loop between helices I and II. PROCHECK analysis indicates an overall $\mathrm{G}$ factor of -0.23 and only two bad contacts. Table 1 also lists other relevant refinement statistics, all of which indicate reasonable geometry and energetics for the final ensemble and average-minimized structures.

\section{Description of the B. subtilis ACP NMR-Derived Structures}

The solution structure of the $B$. subtilis ACP consists of a four a-helical bundle where helices I (D2-L15, colored red in Figures 1a and 1b), II (S36-E49, colored green in Figures 1a and 1b), and IV (V65-Q75, colored magenta in Figures $1 a$ and $1 b$ ) are essentially parallel and have an approximately equal length (11-14 residues) with an updown-down topology. Helix III (D56-K61, colored orange in Figures $1 \mathrm{a}$ and $1 \mathrm{~b}$ ) is shorter relative to the other helices and packs effectively perpendicular to the helical bundle. Three different length loops (colored blue) connect the four helices. A long loop (G16-D35) connects helix I to helix II. A shorter loop (F50-S55) connects helix II to III, and a 3-residue loop (I62-T64) links helix III and VI. The a helices I, II, and IV of $B$. subtilis ACP have an amphipathic character. Many of the long-range NOEs involved in packing of the helical bundle occur between the hydrophobic side chains, corresponding to residues L4, V7, I11, and L15 in a I; V39, V40, L42, V43, and L46 in a II; and V65, A68, V69, and I72 in a VI. These residues form the hydrophobic core of ACP, in which the side chain interactions between these residues stabilize the global fold of the protein. The $B$. subtilis ACP has two phenylalanines, F28 and F50, that are located in the second half of the first long loop and correspond to the first residue of the second loop, respectively. In the average-minimized structure, both F28 and F50 interact with the hydrophobic core that is formed by the packing of the helical bundle. F28 and F50 are in close contact with residues I10, I11, and L15 in a I; V39, L42, and L46 in a II; and $\mathrm{V} 65$ and 172 in a $\mathrm{VI}$, which is consistent with the experimental long-range NOEs. Conversely, the side chains of many hydrophilic residues in the helical bundle are solvent exposed. These residues correspond to E5, T8, K9, and D13 in a I; D38, E41, E45, E47, and D48 in a II; and D67, N70, Y71, N74, and Q75 in a IV.

Relative to the other helices, $\alpha$ III is highly hydrophilic and contains only 1 hydrophobic residue, A59. Given its high rmsd relative to the helix bundle, a III could be 


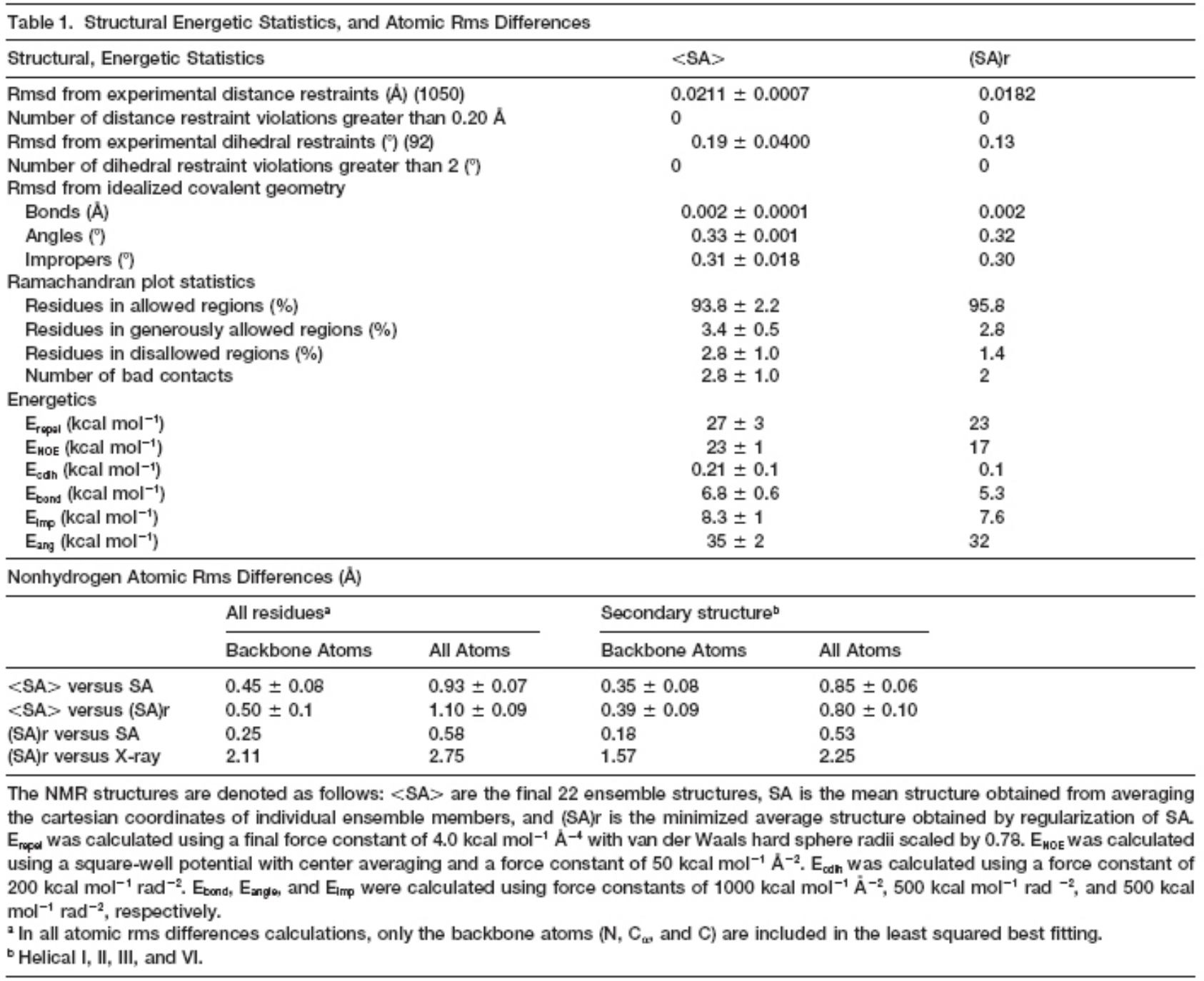

viewed as part as of the loop region that connects helix II and IV. In fact, this short helix is missing in the structures of the other members of the ACP family [12 and 13]. In the $B$. subtilis ACP structure, long-range NOEs that are consistent with the ACP three-dimensional structure indicate that A59 is in close contact with residues V40 in a II and V65 in a VI. This indicates that A59 participates in a hydrophobic interaction with the helical bundle. Additionally, D58 is in close proximity with $\mathrm{Y} 71$ from a VI, where the phenolic hydroxyl might form a hydrogen bond with the carboxyl side chain from D58. These interactions define the close contact of helix III with both helices II and VI and determine its perpendicular orientation $\left(\sim 60^{\circ}\right)$.

The long loop between helices I and II is composed of 20 residues (G16-D35) and starts with a distorted short turn (G16-A20) that is defined by numerous medium-range (i,i+2 and $i, i+3)$ interactions. The remainder of this long loop is not well defined and effectively adopts an unstructured random conformation that is a result of a deficiency of medium- and long-range NOE restraints. The lack of a defined structure for most of the long loop region is also made apparent by an absence of slow-exchanging amide protons. As a result, the rmsd for this loop region is much higher $(0.57 \AA$ and $1.25 \AA$ ) for backbone and all atoms, respectively, than the rmsd for secondary structure regions (0.30 $\AA$ and $0.80 \AA)$. The long loop has an amphipathic characteristic that contains 10 hydrophobic and 10 hydrophilic residues. The $B$. subtilis ACP structure indicates that most of the hydrophilic residues are exposed to the solvent, while most of the hydrophobic residues are orientated toward the central hydrophobic helical core. In addition to the long-range interactions described previously for F28, a number of long-range NOEs were observed between the hydrophobic side chains from the long loop region and both the C-terminal end of helix I and the $\mathrm{N}$-terminal end of helix $\mathrm{VI}$.

\section{Holo and Apo Forms of B. subtilis ACP}

The NMR sample used for the $B$. subtilis ACP structure determination consists of an approximate 60:40 mixture of the holo and apo forms, respectively. The 4'-PP moiety from CoA is attached to the conserved S36 on ACP, as evident by the presence of two distinct sets of chemical shifts in the $1 \mathrm{H}-{ }^{15} \mathrm{~N} \mathrm{HSQC} \mathrm{spectrum} \mathrm{for} \mathrm{the} \mathrm{N-termi-}$ nal region of helix II that is in the vicinity of S36 (Figure 2). Figure $2 a$ illustrates the distinct chemical shifts that are observed in the $1 \mathrm{H}-{ }^{15} \mathrm{~N} \mathrm{HSQC}$ for the residues in the vicinity of the 4'-PP prosthetic group. For example, the am- 

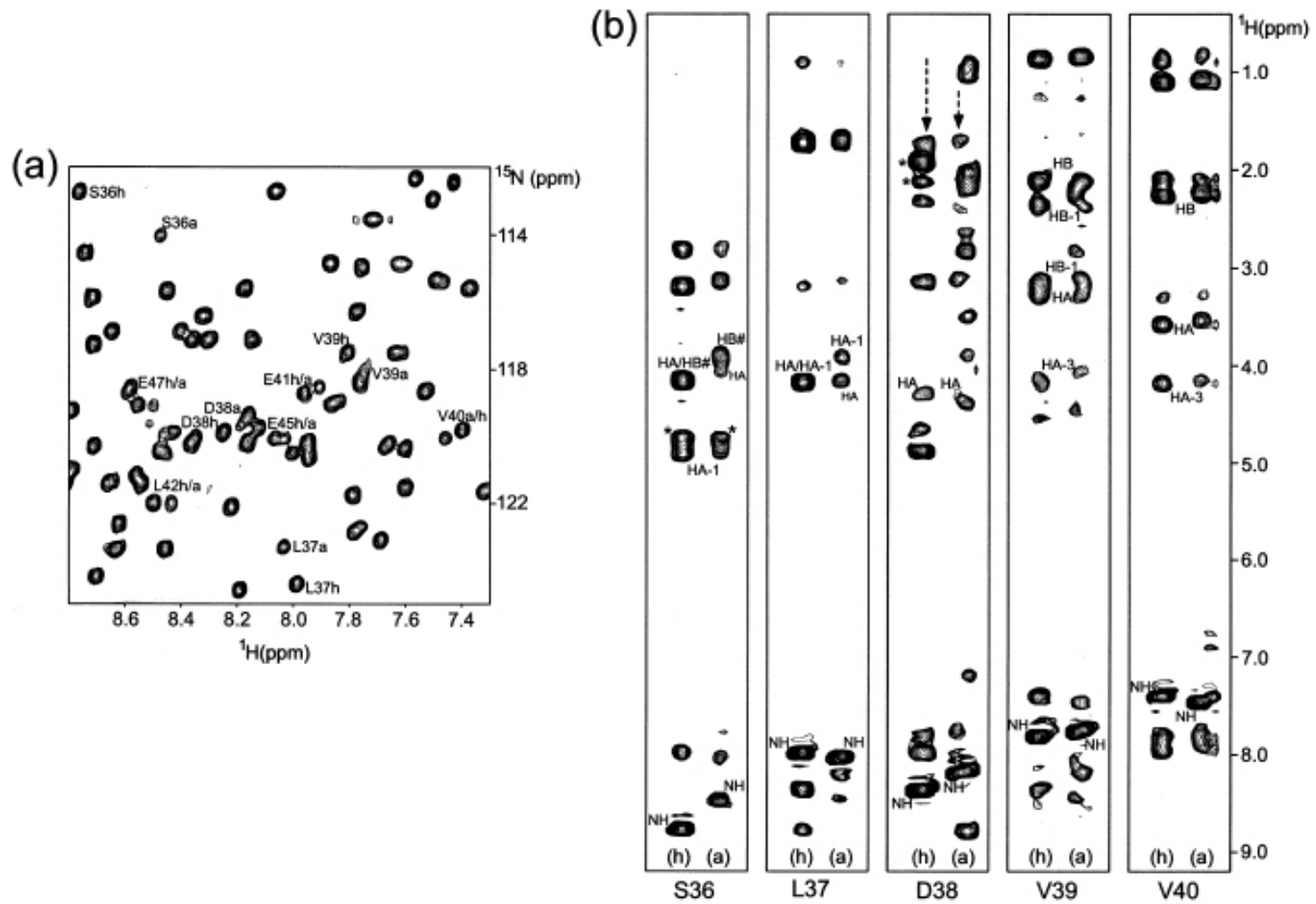

Figure 2. 2-D $1 \mathrm{H}-{ }^{15} \mathrm{~N}$ HSQC and 3-D ${ }^{15} \mathrm{~N}$-edited NOESY-HMQC Spectrum of Apo and Holo Forms of $B$. subtilis ACP

(a) An expansion of the $2-\mathrm{D} 1 \mathrm{H}-{ }^{15} \mathrm{~N}$ HSQC spectrum, illustrating residues that exhibit distinct ${ }^{15} \mathrm{~N} / \mathrm{NH}$ chemical shifts in the apo and holo forms of B. subtilis ACP.

(b) Composite of amide strips taken from the 3-D ${ }^{15} \mathrm{~N}$-edited NOESY-HMQC spectrum of $B$. subtilis ACP, corresponding to residues S36-V40 that exhibit distinct ${ }^{15} \mathrm{~N} / \mathrm{NH}$ chemical shifts in the apo and holo forms of $B$. subtilis ACP. For clarity, some of the crosspeak assignments are labeled. $\mathrm{HDO}$ and cross-peaks that are attributed to other residues are labeled with an asterisk

ide proton and ${ }^{15} \mathrm{~N}$ chemical shifts of S36 are at $8.76 \mathrm{ppm}$ and $112.7 \mathrm{ppm}$, respectively, for holo-ACP compared to 8.47 ppm and 114.0 ppm for apo-ACP. Similarly, L37 exhibits amide proton and ${ }^{15} \mathrm{~N}$ chemical shifts at $7.99 \mathrm{ppm}$ and $124.4 \mathrm{ppm}$ for holo-ACP, compared to $8.03 \mathrm{ppm}$ and $123.3 \mathrm{ppm}$ for apo-ACP. D38 exhibits amide proton and ${ }^{15} \mathrm{~N}$ chemical shifts at $8.34 \mathrm{ppm}$ and $120.0 \mathrm{ppm}$ for holoACP, compared to $8.17 \mathrm{ppm}$ and $119.7 \mathrm{ppm}$ for apo-ACP. The residues that incur a chemical shift change as a function of the holo and apo forms of ACP are colored in Figure $1 \mathrm{c}$, where $S 36$ is colored magenta, and the other perturbed residues are colored red.
Despite the observed chemical shift perturbations in the $1 \mathrm{H}-{ }^{15} \mathrm{~N}$ HSQC spectra between the holo and apo forms of $\mathrm{ACP}$, the ${ }^{13} \mathrm{C}$ and $1 \mathrm{H}$ side chain chemical shifts for these residues are essentially unchanged. This was apparent from the HSQC-based triple-resonance experiments, in which a doubling of peaks was observed based on the different amide proton and ${ }^{15} \mathrm{~N}$ resonances for the apo and holo forms, but the remainder of the crosspeaks were identical or nearly identical. Additionally, the ${ }^{15} \mathrm{~N}$-edited NOESY spectra did not show any distinct NOE patterns for holo- and apo-ACP residues (Figure $2 b$ ). This was also the case for S36, which has the largest chemical shift difference in the $1 \mathrm{H}-{ }^{15} \mathrm{~N} H S Q C$
Figure 3. Sequence Alignment of E. coli and act apoACP with $B$. subtilis ACPIdentical residue types are colored black, and identical residue classes are colored red

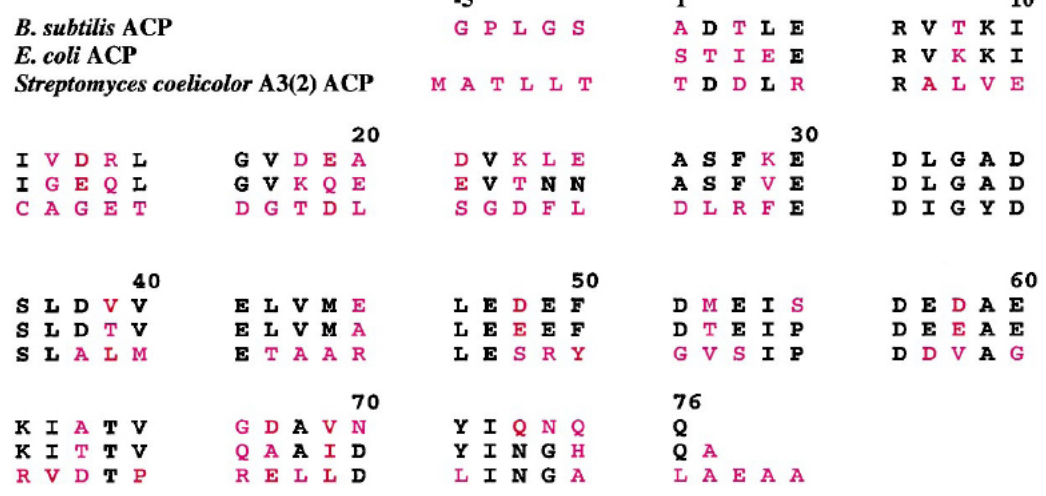


spectra. For holo-ACP, no additional NOEs were observed between the 4'-PP prosthetic group and the protein itself, except for the linkage between the 4'-PP prosthetic group and S36. These results suggest that the structures of holoACP and apo-ACP are essentially the same and that the 4'PP prosthetic group of holo-ACP does not incur any preferential interactions with the protein. This implies that either the 4'-PP prosthetic group is essentially solvent exposed or experiences rapid exchange between multiple low- affinity binding interactions with ACP. Because the function of ACP is to carry the fatty acid chain as it is elongated by FAS, it is not surprising that the 4'-PP prosthetic group is readily accessible at the protein surface. S36 is located at the top of helix II at the surface of the protein, making it readily accessible by ACPS and FAS (Figure 1c). Proximal to S36 is a hydrophobic pocket, composed of residues F28, V39, L42, V43, L46, and I62, which may be involved in binding the acyl chain as it is lengthened (Figure 1d).

The addition of acyl moieties to ACP results in stabilizing the protein against $\mathrm{pH}$ denaturation. There is a direct correlation between the degree of protection from $\mathrm{pH}$ denaturation and the increasing length of the acyl chain [20 and 21]. Additionally, the binding affinity of acyl-ACP to octyl-sepharose based on the length of the acyl chain and the lack of affinity for apo-ACP suggests the presence of an acyl chain binding site on ACP [22]. Furthermore, 1D 19F$1 \mathrm{H}$ cross-relaxation and chemical shift perturbation experiments have identified a number of potential ACP residues that may be involved in the interaction of the acyl chain or a conformational change in ACP that occurs upon acylation [23 and 24]. Again, the extent of the observed chemical shift changes correlates with an increase in the acyl chain length. Finally, the X-ray structure of ACP complexed with ACPS demonstrates that the 4'-PP prosthetic group is embedded in the hydrophobic pocket of ACP [25]. These results, taken in total, support the proposition that the growing acyl chain that is attached to ACP binds in a hydrophobic pocket that is proximal to S36. The affinity of the acyl chain for the hydrophobic pocket increases as a function of increasing chain length. This follows the expected trend that as the chain length increases and becomes more hydrophobic in nature, there is a greater tendency for the acyl chain to avoid the solvent. In the case of the NMR structure of $B$. subtilis ACP reported here, only the 4'-PP prosthetic group is attached to ACP. This differs from the studies reported above, excluding the X-ray structure, in which the acyl chains contained anywhere from 2 to $>10$ additional carbon atoms relative to 4'-PP. The 4'-PP prosthetic group itself is not extensively hydrophobic, and, as evident from the results described above, increasing the acyl chain length has a pronounced effect on hydrophobicity. Therefore, it is not too surprising that the NMR structure does not identify that the 4'-PP binds exclusively into the hydrophobic pocket, and that the apo- and holo-ACP structures are essentially identical.

\section{Comparison of the B. subtilis ACP Structure with the E. coli ACP and act apo-ACP Structures}

NMR structures for E. coli ACP and act apo-ACP were reported in the literature [12, 13 and 15] prior to initiation of our efforts on the structure determination of $B$. subtilis ACP. Amino acid sequence alignments indicate that $E$. coli ACP is highly homologous to $B$. subtilis ACP, where 53 of 76 residues correspond to identical residue classes (70\%) (Figure 3). Similarly, comparison of act apo-ACP with $B$. subtilis ACP indicates that 38 of 76 residues correspond to identical residue classes (50\%) (Figure 3 ). The overall sequence homology suggests that the three proteins should have a similar global fold (Figures $4 a$ and $4 b$ ).

Comparison of the published structures for $E$. coli ACP (PDB ID:1ACP) and act apo-ACP (PDB ID:2AF8) with $B$. subtilis ACP indicate that the three proteins have similar secondary structure elements, where the a-helical content corresponds to $51 \%, 49 \%$, and $60 \%$ for E. coli, act apo-, and $B$. subtilis ACP, respectively. The overall ACP structure consists of a four a-helical bundle where three a helices are relatively long (6-15 residues) and one helix is short (0-6 residues). Despite the similarity in the amino acid sequence and secondary structure features, the global fold for the three ACP structures is distinct. This is made readily apparent by the superposition of the average-minimized structures for E. coli ACP and act apoACP with $B$. subtilis ACP (Figures $4 a$ and $4 b$ ). The atomic rmsd of the $\mathrm{Ca}$ atoms between $E$. coli and $B$. subtilis ACP for the 53 homologous residues is $4.63 \AA$. The comparison improves to $3.82 \AA$ for similar secondary structure regions. Similarly, the atomic rmsd of the $\mathrm{Ca}$ atoms between act apo- and B. subtilis ACP for the 38 homologous residues is $4.39 \AA$. Again, an improvement to $2.66 \AA$ occurs when only similar secondary structure regions are used in the comparison. Moreover, the rmsd between the $\mathrm{Ca}$ atoms for the 36 identical residue classes between $E$. coli and act apo is $5.60 \AA$, which is much higher than the deviations between these two proteins and $B$. subtilis ACP. These large rmsd indicate that the three ACP structures are relatively unique.

The observed large rmsd between the three ACPS structures are located mainly in the short a helix III and the long loop region between helix I and II. The short a helix III is not present in the average-minimized act apo-ACP structure. Also, in some models, the short a III is absent from the ensemble of structures for both E. coli and act apo-ACP. Some of the observed differences between the $B$. subtilis ACP structure and both the E. coli and act apo-ACP structures result from unusual features of the $E$. coli and act apo-ACP structures. An example is the presence of a large kink in a helix I for $E$. coli ACP that results in this helix being extremely distorted. An additional factor contributing to the difference between $B$. subtilis ACP structure and both the E. coli and act apo-ACP is attributed to the relative displacement of the helical regions. The observation of distinct structures for the three ACP proteins is unexpected given the reasonable sequence homology and the obvious fact that the proteins are functionally identical. During the time frame that the three structures for ACP have been determined, there have been advances in NMR methodology in its application in solving solution structures of protein. Therefore, a potential source for the structural difference may have resulted from limitations in the structure determination process for the early ACP structures instead of an intrinsic difference in the proteins. Furthermore, physi- 
cal behavior encountered with the previous ACP structures that were not a factor in the $B$. subtilis ACP structure would have only exasperated the methodology limitations for the earlier structures.

The NMR analysis of the E. coli ACP structure suggests the existence of equilibrium between multiple conformers [13 and 14]. Additional technical difficulties were reported in the structure determination of spinach ACP and the nodulation protein NodF from Rhizobium leguminosarum, in which both proteins share homology with ACP [26 and 27]. In both cases, only low-resolution structures were determined, because of the lack of experimental restraints and the fact that three-dimensional structures were not released to the Protein Data Bank. Nevertheless, the overall fold and secondary structure of spinach ACP and the nodulation protein NodF were consistent with the E. coli ACP structure. These results clearly suggest an inherent technical difficulty that was encountered with the previous ACP structures that was not a factor in the $B$. subtilis ACP structure.

The structure determinations for both $E$. coli and act apo-ACP were limited to homonuclear two-dimensional NMR experiments. In the case of the B. subtilis ACP structure, we were able to take full advantage of the current state of multidimensional heteronuclear NMR methodologies utilizing ${ }^{15} \mathrm{~N} /{ }^{13} \mathrm{C}$ isotope-enriched $B$. subtilis ACP [28]. This approach was not readily accessible for the determination of the E. coli and act apo-ACP structures. Further complicating the inherent limitations in the analysis of 2-D NMR data is the helical nature of ACP, causing the NMR spectra to exhibit relatively narrow chemical shift dispersion. As a result of these relative limitations, the structures for $E$. coli and act apo- ACP were based on a minimal number of distance restraints, especially long-range distance restraints, compared to those used in the determination of the B. subtilis ACP structure. The E. coli ACP (77 residues) structure was based on a total of 478 distance restraints comprising $30 \mathrm{H}$ bond distance restraints; 101 intraresidue distance restraints; and 205 sequential, 87 short-range, and 55 long-range interresidue distance restraints. The average number of distance restraints was only 6.2 restraints per residue. Similarly, the act apo-ACP (86 residues) structure was based on a total of 747 distance restraints comprising $48 \mathrm{H}$ bond distance restraints; 240 intraresidue distance restraints; and 235 sequential, 131 short-range, and 93 long-range distance restraints. The average number for act apo-ACP was 8.7 restraints per residue. Conversely, the $B$. subtilis ACP (76 residues) structure reported herein was based on a total of 1050 distance restraints, with an average of 13.8 restraints per residue, comprising $54 \mathrm{H}$ bond distance restraints; 337 intraresidue distance restraints; and 231 sequential, 188 short-range, and 240 long-range interresidue restraints. Similarly, the $B$. subtilis ACP structure was based on more $\varphi$ and $\psi$ dihedral angle restraints relative to both $E$. coli and act apo-ACP. A total of $96 \varphi$ and $\psi$ dihedral angle restraints were used for the $B$. subtilis ACP structure, compared to 54 and 63 for the $E$. coli and act apo-ACP structures, respectively. In addition, the $B$. subtilis ACP structure was refined using both $\mathrm{Ca} /$ $C B$ chemical shift restraints [29] and a conformational data

\begin{tabular}{|c|c|c|c|}
\hline & E. coli ACP & act apo-ACP & B. subtilis ACP \\
\hline \multicolumn{4}{|l|}{ All Residues } \\
\hline Backbone & 2.3 & 1.47 & 0.45 \\
\hline All atoms & 3.3 & 1.84 & 0.93 \\
\hline \multicolumn{4}{|c|}{ Secondary Structure } \\
\hline Backbone & & 1.01 & 0.35 \\
\hline All atoms & & 1.45 & 0.85 \\
\hline
\end{tabular}

${ }^{a}$ The NMR ensemble for the E. coli, act apo-, and B. subtilis ACP structures consist of 7,24 , and 22 , respectively.

base potential [30], which were not available refinement approaches for the E. coli and act apo-ACP structures. Clearly, the increase in the number of the restraints for the $B$. subtilis ACP structure was attributed to the utilization of ${ }^{13} \mathrm{C} /{ }^{15} \mathrm{~N}$-labeled ACP in combination with 3-D heteronuclear NMR experiments. This approach overcomes most of the limitations encountered with the unlabeled ACP samples and 2-D based NMR experiments.

It has been well-established that the accuracy and precision of NMR solution structures is correlated with the number of structural restraints [31]. In addition to the number of restraints, the quality of the ACP structures is also reflected by the rmsd between each structure in the ensemble relative to the average structure. Typically, a highresolution NMR structure exhibits a backbone rmsd of $<0.5$ $\AA$. As apparent in Table 2, the structures for E. coli and act apo-ACP have extremely high rmsd values, suggestive of a low-resolution structure, whereas, the $B$. subtilis ACP structure reported herein demonstrates rmsd values consistent with a high-quality structure. Again, these differences presumably reflect the inherent limitations of 2-D homonuclear NMR methodologies compared to multidimensional heteronuclear NMR approaches and the resulting increase in the number of restraints needed to solve the $B$. subtilis ACP structure.

Recently, the solution structure of a peptidyl carrier protein (PCP) was published [32]. PCP has a similar biological function to ACP and contains a conserved serine where the cofactor 4'-phosphopantetheine is attached. The sequence alignment between PCP and ACP indicates a lack of sequence homology, with the exception of the site of cofactor binding. In addition, the calculated pl values of PCP, E. coli, and act apo-ACP are strikingly different. However, PCP has topology and structural features similar to those of ACP, where the PCP structure is composed of a distorted four $\alpha$ helical bundle with an extended loop between the first two helices. The similarity between the PCP and $B$. subtilis ACP structures supports the general belief that function is a primary factor in defining a protein's structure. Therefore, the observed structural difference between $B$. subtilis ACP with both $E$. coli and act apo-ACP appears to be attributed to limitations in the available NMR methodology for the E. coli and act apo-ACP structures, which was further exasperated by the physical behavior of both $E$. coli and act apoACP. Given the high-sequence homology and the identical functions, it appears unlikely that the observed differences between the three ACP structures reflect an in- 
(a)

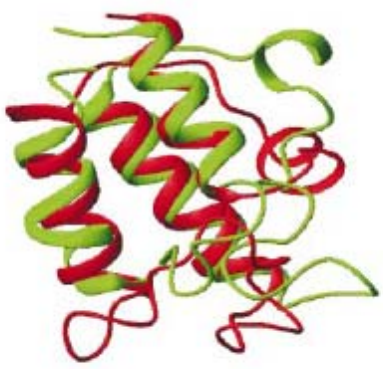

(b)

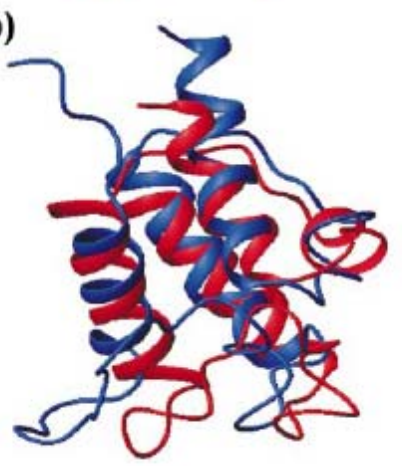

(c)

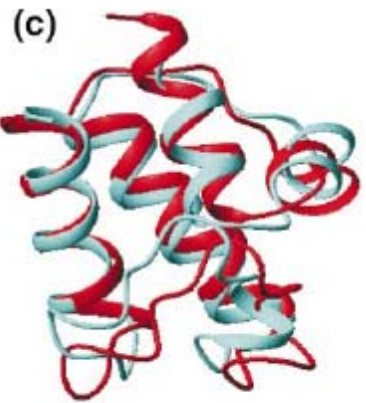

(a)

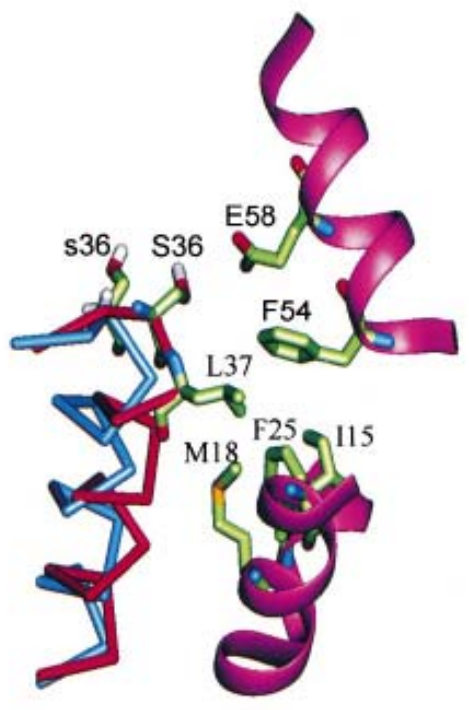

(b)

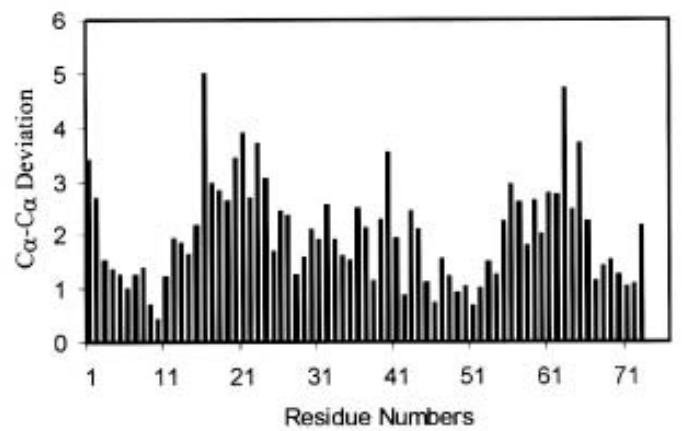

Figure 4. Overlays of the Ribbon Diagrams of $B$. subtilis ACP with the E. coli, act apo, and X-ray ACP Structures

(a) B. subtilis ACP (red) and E. coli ACP (green).

(b) B. subtilis ACP (red) and act apo-ACP (blue).

(c) Free ACP NMR (red) and X-ray ACP in the ACP-ACPS complex (turquoise)

herent difference in the structure of these proteins. The accuracy and precision of the B. subtilis ACP structure is supported by the high-quality structural and energetic statistics and the relatively low atomic rms differences reported in Table 1. Further support for the reliability of the $B$. subtilis ACP structure is the fact that the $B$. subtilis ACP was useful in aiding in the solution of the X-ray structure of ACP in the $B$. subtilis ACP-ACPS complex [25]. Similar attempts to use the E. coli ACP structure to assist the X-ray structure refinement of the ACP-ACPs complex was not successful, presumably a result of the large difference in the E. coli ACP structure.

\section{Comparison of the Structures of Free B. subtilis ACP with ACP in the ACP-ACPS Complex}

An overlay of the ribbon diagrams and $\mathrm{Ca}$ traces of the NMR structure for the free $B$. subtilis ACP and the X-ray structure of ACP from the $B$. subtilis ACP-ACPS complex with bound CoA [25] is illustrated in Figure 4c. The backbone atomic rms difference between the free and bound forms of ACP is $2.11 \AA$ and $1.57 \AA$ for all backbone atoms and regions of defined secondary structure, respectively. Figure $5 \mathrm{~b}$ illustrates the Ca deviations on a per residue basis (A1-Q73) between the free and bound forms of ACP. The observed Ca deviations range from 0.43 to $4.87 \AA$, with significant differences localized in the first long loop and the $\mathrm{N}$ terminus
Figure 5. Structural Differences Between the Free and Bound Forms of $B$. subtilis ACP

(a) The best-fit superposition of the Ca atoms of helix II of free ACP (blue) and bound ACP (red) in the ACP-ACPS complex. The ribbons (pink) are part of helices I, II, and IV from ACPS in the ACP-ACPS complex. Part of the hydrophobic contacts in the ACP-ACPS complex involve L37 from ACP with I15, M18, F25, and F54 from ACPS. S36 from ACP is displaced and closer to E58 from ACPS in the ACP-ACPS complex. S36 in the free ACP structure is $3.6 \AA$ further away from E58 of ACPS in the ACP-ACPS complex.

(b) Ca deviation between the free $B$. subtilis ACP structure and the ACP structure in the ACP-ACPS complex as a function of residue number

of helix II. The long loop in the free ACP structure is less defined and unstructured, a result of the limited number of medium- and long-range distance restraints. Conversely, for ACP in the ACP-ACPS complex, a short a helix is observed for residues F28-D31 in this long loop. This is the first example of a helical conformation within the long loop region for $A C P$, suggesting a decrease in flexibility in the ACP-ACPS complex. Comparison of the three long helices between the free and bound ACP structures indicates that helix VI is shorter in the ACP-ACPS complex (V65Y71, 7 residues) than in the free form (V65-Q75, 11 residues). The $C$ terminus for ACP in the ACP-ACPS complex appears to exhibit an increase in flexibility that is suggested by the shorter helix and the missing electronic densities for the last 3 residues (N74-Q76). 
The X-ray structure of ACP in the ACP-ACPS complex [25] indicates that the major interactions between holo-ACP and ACPS are predominately hydrophilic in nature and occurs between helix I of ACPS and helix II of ACP. A key salt bridge interaction occurs between the side chain carboxyl of both D35 and D38 in ACP and the side chain guanido group of R14 from ACPS. This key salt bridge is in the vicinity of S36 from ACP, which is the location of the attachment of 4'-PP from CoA to ACP by ACPS. Two additional salt bridges, involving residues E41 from ACP and R21 from ACPS and D48 from ACP and both E22 and R24 from ACPS, respectively, are present in the ACP-ACPS interface. The ACP-ACPS binding interaction is additionally stabilized by two hydrophobic contacts, in which L37 and M44 from ACP protrude into hydrophobic pockets on ACPS, as shown in Figure 5a. L37 and M44 from ACP extend into the ACPS pockets formed by residues M18, F25, F54, and I15 and residues F25, R28, and Q22, respectively. The effect of these interactions between ACP and ACPS is the relative displacement of helix II between the bound ( Figure 5 , red) and free ( Figure 5, blue) forms of ACP, where helix II is forced closer to ACPS in the ACP-ACPS complex. Moreover, the displacement of the N-terminal end of helix II probably allows for S36 of ACP to be pulled into the ACPS active site for the attachment of 4'-PP from CoA. E58 is a highly conserved residue in ACPS that is involved in coordinating the calcium that is bound to CoA. The side chain for S36 from ACP is in close contact with the side chain of E58 from ACPS in the ACP-ACPS complex. The close proximity of S36 from ACP to E58 from ACPS is probably required for the transfer of the 4'-PP moiety from CoA to ACP. Superimposing the free ACP structure on the bound ACP structure in the ACP-ACPS complex indicated that the side chain for $S 36$ from free ACP is $\sim 3.6 \AA$ further from the E58 side chain of ACPS than in the complex. In the ACPACPS complex containing bound CoA, the dipole of helix II in ACP is pointing toward the pyrophosphate moiety of CoA, which orients S36 near the ß-phosphate of CoA and an adjacent water molecule [25]. This orientation of helix II presumably initiates the transfer of the 4'-PP from CoA to ACP. This then implies that the reorganization of helix II from ACP upon complex formation with ACPS is a necessary component of the mechanism to transfer the 4'-PP moiety from CoA to ACP.

\section{Biological Implications}

Acyl carrier protein (ACP) is an essential component in the biosynthesis of fatty acids and other biosynthetic pathways that require acyl transfer steps. The function of ACP is to carry the fatty acid chain as it is elongated by FAS [1, 2 and 3]. ACP is converted to its active holo form by holo-acyl carier protein synthase (ACPS) with the transfer of 4'-phosphopantetheine from coenzyme $A$ to a conserved serine (S36) on ACP. The extension of the fatty acid chain then occurs through a linkage to the terminal thiol of 4'-PP attached to ACP. ACP and ACPS have been demonstrated to be critical to the viability of $E$. coli [10 and 11], suggesting that the activities of ACP and ACPS are potential targets for the devel- opment of novel antibiotics. A detailed understanding of the structural contribution of ACP and ACPS to the activity of both proteins is fundamental to a drug design program.

Comparison of the $B$. subtilis ACP structure with both the E. coli and act apo-ACP structures indicates a significant discrepancy between the three ACP structures. Because of the identical function and high-sequence homology between the proteins, we do not believe that there is an inherent difference in the ACP structures. Instead, the evidence suggests that the discrepancy is a result of technical difficulties combined with the inherent limitations of the NMR methodologies available to determine the E. coli and act apo-ACP structures. This was not an issue in the structure determination of $B$. subtillis ACP. The application of NMR to solve pro-tein structures has continued to evolve during the time period in which the original ACP structures were determined [28]. The utilization of the current state of multidimensional heteronuclear NMR techniques in conjunction with ${ }^{13} \mathrm{C}$ and ${ }^{15} \mathrm{~N}$ isotope enrichment permitted the determination of a high-resolution structure for $B$. subtilis $A C P$, as evident by the structural, energetic, and rmsd statistics (Table 1). We believe that the high-quality $B$. subtilis ACP structure provides an accurate description of the structure of ACP.

Comparison of the free ACP structure with the structure of ACP in the ACP-ACPS complex, and comparison of the apo-ACP structure with the holo-ACP structure may provide some insight into the mechanism of ACP activation by ACPS. The addition of 4'-PP to ACP does not result in any observable difference in the structure of the apo and holo forms of the protein. Furthermore, there is no evidence for any interaction between 4'-PP and ACP, except for the attachment at S36. This implies that 4'-PP in ACP is readily accessible by FAS for elongation of the fatty acid chain. Proximal to $S 36$ is a hydrophobic pocket that may be involved in binding the acyl chain as it is lengthened and becomes more hydrophobic (Figure 1d). The lack of a structural difference between holo- and apo-ACP also suggests that the dissociation of holo-ACP from the ACP-ACPS complex is not facilitated by a structural change in ACP that is induced by the attachment of 4'-PP. Nevertheless, ACPS does induce a significant perturbation in the ACP structure upon complex formation. The result is a displacement of helix II and the appropriate positioning of S36 from ACP to enable the transfer of 4'-PP from CoA to ACP. S36 from ACP is effectively drawn into the ACPS structure into close proximity to CoA in ACPS, with the alignment of the helix II dipole with the S36-4'-PP attachment point initiating the transfer [25]. After transfer of the 4'-PP moiety from ACPS to ACP occurs, dissociation of the ACP-ACPS complex is probably facilitated by relaxation of the bound structure of $\mathrm{ACP}$ to its free structure conformation.

\section{Experimental Procedures}

\section{B. subtilis ACP Sample Preparation}

The uniform ${ }^{15} \mathrm{~N} /{ }^{13} \mathrm{C}$-labeled $B$. subtilis ACP (81 residues) was cloned into a pGEX-6P-1 vector and expressed in E. coli strain BL21DE3 (pLysS). Cells were grown at $37^{\circ} \mathrm{C}$ on minimal medium containing $\left[{ }^{15} \mathrm{~N}\right]$ ammonium sulfate and $\left[\mathrm{U}-{ }^{13} \mathrm{C}\right]$ glucose. For purification, $20 \mathrm{~g}$ of 
the above cell pellet was resuspended in $300 \mathrm{ml}$ breaking buffer consisting of $50 \mathrm{mM}$ Tris- $\mathrm{Cl}$ ( $\mathrm{pH} 8.0$ ), $300 \mathrm{mM} \mathrm{NaCl}, 10 \mathrm{mM} \mathrm{MgCl}_{2}$, and $2 \mathrm{mM}$ freshly prepared $\mathrm{MnCl}_{2}$. Protease inhibitor tablets (Boehringer Mannheim $\mathrm{GmbH}$ ), RNaseH and Dnasel, (Sigma Chemical Company) were added to the solution to prevent protease activity and to decrease viscosity of the solution. The cells were lysed by three passages through a microfluidizer and centrifuged at $15,000 \times \mathrm{g}$ for 20 $\min$ at $4^{\circ} \mathrm{C}$ to remove the cell debris. Glutathione sepharose $4 \mathrm{~B}$ resin (Amersham Pharmacia Biotech) equilibrated with the same breaking buffer was added to the clear supernatent. The mixture was incubated at $4^{\circ} \mathrm{C}$ for $1 \mathrm{hr}$ prior to packing the resin into a suitable column. The column was washed, and the GST-ACP was eluted with a buffer containing $50 \mathrm{mM}$ Tris- $\mathrm{Cl}$ ( $\mathrm{pH}$ 8.0), $10 \mathrm{mM} \mathrm{MgCl}_{2}, 5 \mathrm{mM}$ DTT, and 60 $\mathrm{mM}$ reduced glutathione. The resulting GST-ACP solution was dialyzed overnight against $50 \mathrm{mM}$ Tris- $\mathrm{Cl}$ ( $\mathrm{pH} \mathrm{8.0),} 150 \mathrm{mM} \mathrm{NaCl}, 1 \mathrm{mM}$ EDTA, and $1 \mathrm{mM}$ DTT. The fusion protein was cleaved with Prescission Protease (Amersham Pharmacia Biotech) at room temperature for $3 \mathrm{hr}$ at a ratio of $1 \mathrm{U}$ enzyme per $500 \mu \mathrm{g}$ protein. The resulting protein mixture was loaded on a MonoQ HR16/10 column (Amersham Pharmacia Biotech), and the ACP protein was eluted with a $\mathrm{NaCl}$ gradient. ACP containing fractions were pooled and loaded on a TSK G2000 size exclusion column prior to the NMR sample preparation.

\section{NMR Data Collection and Assignments}

The NMR sample is a mixture of ${ }^{15} \mathrm{~N} /{ }^{13} \mathrm{C}$-double-labeled apo- and holo-ACP in $50 \mathrm{mM}$ Bis-Tris (pH 6.4), $100 \mathrm{mM} \mathrm{NaCl}, 10 \mathrm{mM} \mathrm{MgCl}$, and $10 \mathrm{mM}$ DTT with $0.02 \% \mathrm{NaN}_{3}$ in $5 \% \mathrm{D} 2 \mathrm{O} / 95 \% \mathrm{H}_{2} \mathrm{O}$ solution. The protein concentration was approximately $1 \mathrm{mM}$.

All spectra were recorded at $25^{\circ} \mathrm{C}$ on a Varian Unity+ 600 spectrometer equipped with a triple-resonance $1 \mathrm{H} /{ }^{13} \mathrm{C} /{ }^{15} \mathrm{~N}$ probe and an actively shielded $z$ gradient-pulsed field accessories. Two-dimensional $1 \mathrm{H}-{ }^{15} \mathrm{~N}$ HSQC and all HSQC-based 3-D ${ }^{15} \mathrm{~N}$-edited NOESY and 3-D triple-resonance experiments were recorded with the enhanced-sensitivity-pulsed field gradient approach [33]. This approach provides coherence transfer selection both to improve sensitivity and to eliminate artifacts as well as for solvent suppression. For the $2-\mathrm{D} 1 \mathrm{H}-{ }^{15} \mathrm{~N}$ HSQC and the $3-\mathrm{D}{ }^{15} \mathrm{~N}$-edited NOESY experiments, hard ${ }^{13} \mathrm{C} 180^{\circ}$ pulses were applied in the middle of the $\mathrm{t} 1$ period to dephase ${ }^{13} \mathrm{C}$ polarization during the transfer of magnetization between the proton and the ${ }^{15} \mathrm{~N}$ nuclei. The simultaneous ${ }^{15} \mathrm{~N} /{ }^{13} \mathrm{C}$-edited NOESY experiment with separation via the carbon and nitrogen of the destination sites was performed for simultaneous identification of NOEs from both amides and aliphatic protons [34 and 35].

Data sets were typically processed and displayed on an SGI workstation with the program packages NMRDraw and NMRPipe [36]. A skewed $60^{\circ}$ phase-shifted sine-bell function and a single zero-filling were used in each dimension prior to Fourier transformation. For triple-resonance 3-D experiments, the time domain was extended by a factor of two, prior to zero-filling, using froward-backward linear prediction in the ${ }^{15} \mathrm{~N}$ (t2) dimension where mirror image linear prediction was used for constant-time $1 \mathrm{H}^{-13} \mathrm{C}$ correlation experiments [37]. The programs PIPP and STAPP were used for data analysis and semiautomatic assignments [38].

The complete sequential and side chain assignments (>95\%) of the $1 \mathrm{H},{ }^{15} \mathrm{~N}$, and ${ }^{13} \mathrm{C}$ resonances were based on the following experiments: $\mathrm{CBCA}(\mathrm{CO}) \mathrm{NNH}, \mathrm{HNCACB}, \mathrm{C}(\mathrm{CC}) \mathrm{TOCSY} \mathrm{NNH}$, $\mathrm{H}(\mathrm{CC}) \mathrm{TOCSY} \mathrm{NNH}$, and HAHB(CBCACO)NN [33 and 39]. Combination of a $2-\mathrm{D}$ constant time ${ }^{13} \mathrm{C}-1 \mathrm{H}$ HSQC experiment optimized for methyls and a 2-D- methyl relay experiment were used for auxiliary methyl assignments of isoleucine, valine, and leucine, as well as methionine residues. Some ambiguous resonances and sequence assignments were further confirmed by sequential NOEs observed in the ${ }^{15} \mathrm{~N}$-edited NOESY experiments [40] and simultaneous ${ }^{15} \mathrm{~N} /{ }^{13} \mathrm{C}$ edited NOESY experiments [34 and 35]. A table of chemical shifts has been deposited in the RCSB Protein Data Bank (PDB ID code 1HY8).

\section{Structure Calculations}

The NMR solution structure is based on interproton distance restraints converted from observed NOEs in both the ${ }^{15} \mathrm{~N}$-edited NOESY [40] and simultaneous ${ }^{15} \mathrm{~N} /{ }^{13} \mathrm{C}$-edited NOESY experiments [34 and 35]. The NOE intensities were classified as either strong (1.8- $2.7 \AA$ ), medium (1.8-3.3 $\AA$ ), or weak (1.8-5.5 $\AA$ ) restraints. The upper distance limits for distances involving methyl protons and nonstereospecifically assigned methylene and methyl protons were corrected appropriately for center averaging [41], and an additional $0.5 \AA$ was added to the upper distance limits for NOEs involving methyl protons averaging [42 and 43]. $\varphi$ and $\psi$ torsion angle restraints were obtained from ${ }^{15} \mathrm{~N}, \mathrm{Ha}$, $C$, and $C B$ chemical shifts using the TALOS program, in which only "good" predictions were used to generate a restraint [16].

The ACP polypeptide chain used for the NMR structural analysis contains 81 residues, including 5 nonnative residues (GPLGS) at the $N$ terminus. The $5 \mathrm{~N}$-terminal residues were not used in the structure calculation since these residues are essentially unstructured and the proline residue exhibits cis-trans isomerization. The structure of $B$. subtilis ACP was determined from a total of 1050 distance restraints comprising 337 intraresidue, 231 sequential, 188 medium, and 240 long- range distance restraints; 54 hydrogen bond restraints; and 92 torsion angles constraints comprised of $46 \varphi$ and $46 \psi$ dihedral restraints. The hydrogen bond restraints were based on the observation of slow-exchanging $\mathrm{NH}$ protons monitored by $2-\mathrm{D} 1 \mathrm{H}-{ }^{15} \mathrm{~N}$ HSQC spectra collected from $5 \mathrm{~min}$ to $4 \mathrm{hr}$ after dissolving the protein in a $\mathrm{D}_{2} \mathrm{O}$ solution.

The structures were calculated using the hybrid distance geometry-dynamical-simulated annealing method of Nilges et al. [44], with minor modifications [45] using the program XPLOR [46], which is adapted to incorporate pseudopotential secondary ${ }^{13} \mathrm{Ca} /{ }^{13} \mathrm{CB}$ chemical shift restraints [29] and a conformational database potential [30 and 47]. The target function that is minimized during constrained minimization and simulated annealing comprises only quadratic harmonic terms for covalent geometry and secondary ${ }^{13} \mathrm{Ca} /{ }^{13} \mathrm{C} \beta$ chemical shift restraints, square-well quadratic potentials for the experimental distance and torsion angle restraints, and a quartic van der Waals term for nonbonded contacts. All peptide bonds were constrained to be planar and trans. There were no hydrogen bonding, electrostatic, or 6-12 Lennard-Jones empirical potential energy terms in the target function. A family of 36 embedded substructures was generated using distance geometry, followed by simulated annealing, regularization, and refinement. An iterative procedure was used to successively introduce an increasing number of NOE distance restraints. A final ensemble of 22 structures contained no distance restraint violations greater than 0.2 $\AA$, and no torsion angle restraint violations greater than $2^{\circ}$ were obtained.

Structural alignment and atomic rmsd comparison between $B$. subtilis ACP, E. coli, and act apo-ACP were determined using MolMol [48], XPLOR [46], and QUANTA (Molecular Simulations).

\section{Acknowledgements}

We would like to thank Kevin Parris and William Somers for allowing us access to the coordinates of the X-ray structure of the ACP-ACPS complex. We would also like to thank Karl Malakian and Michelle Catino for the ACP grown in E. coli. We would also like to thank Mark Stahl for helpful discussions.

\section{References}

1. S.J. Wakil, J.K. Stoops and V.C. Joshi, Fatty acid synthesis and its regulation. Annu. Rev. Biochem. 52 (1983), pp. 537-579.

2. P.W. Majerus and P.R. Vagelos, Fatty acid biosynthesis and the role of the acyl carrier protein. Advan. Lipid Res. 5 (1967), pp. 133.

3. D.J. Prescott and P.R. Vagelos, Acyl carrier protein. Advan. Enzymol. Relat. Areas Mol. Biol. 36 (1972), pp. 269-311. 
4. C.O. Rock and J.E. Cronan, Escherichia coli as a model for the regulation of dissociable (type II) fatty acid biosynthesis. Biochem. Biophys. Acta 1302 (1996), pp. 1-16.

5. B. Shen, R.G. Summers, H. Gramajo, M.J. Bibb and C.R. Hutchinson, Purification and characterization of the acyl carrier protein of the Streptomyces glaucescens tetracenomycin C polyketide synthase. J. Bacteriol. 174 (1992), pp. 3818-3821.

6. J.E. Baldwin, J.W. Bird, R.A. Field, N.M. O'Callaghan, C.J. Schofield and A.C. Willis, Isolation and partial characterization of ACV synthetase from Cephalosporium acremonium and Streptomyces clavuligerus: evidence for the presence of phosphopantothenate in ACV synthetase. J. Antibiot. 44 (1991), pp. 241-248.

7. F. Rusnak, M. Sakaitani, D. Drueckhammer, J. Reichert and C.T. Walsh, Biosynthesis of the Escherichia coli siderophore enterobactin: sequence of the entF gene, expression and purification of EntF, and analysis of covalent phosphopantetheine. Biochemistry 30 (1991), pp. 2916-2927.

8. O. Geiger, H.P. Spaink and E.P. Kennedy, Isolation of the Rhizobium leguminosarum NodF nodulation protein: NodF carries a 4'-phosphopantetheine prosthetic group. J. Bacteriol. 173 (1991), pp. 2872-2878.

9. J.P. Issartel, V. Koronakis and C. Hughes, Activation of Escherichia coli prohemolysin to the mature toxin by acyl carrier protein-dependent fatty acylation. Nature 351 (1991), pp. 759-761.

10. H.E. Takiff, T. Baker, T. Copeland, S.M. Chen and D.L. Court, Locating essential Escherichia coli genes by using mini-Tn10 transposons: the pdxJ operon. J. Bacteriol. 174 (1992), pp. 1544-1553.

11. R.H. Lambalot and C.T. Walsh, Cloning, overproduction, and characterization of the Escherichia coli holo-acyl carrier protein synthase. J. Biol. Chem. 270 (1995), pp. 24658-24661.

12. M.P. Crump et al.T.J. Simpson, Solution structure of the actinorhodin polyketide synthase acyl carrier protein from Streptomyces coelicolor A3(2). Biochemistry 36 (1997), pp. 6000-6008.

13. T.A. Holak, S.K. Kearsley, Y. Kim and J.H. Prestegard, Three-dimensional structure of acyl carrier protein determined by NMR pseudoenergy and distance geometry calculations. Biochemistry 27 (1988), pp. 6135-6142.

14. T.A. Holak, M. Nilges, J.H. Prestegard, A.M. Gronenborn and G.M. Clore, Three-dimensional structure of acyl carrier protein in solution determined by nuclear magnetic resonance and the combined use of dynamical simulated annealing and distance geometry. Eur. J. Biochem. 175 (1988), pp. 9-15.

15. T.A. Holak, M. Nilges and H. Oschkinat, Improved strategies for the determination of protein structures from NMR data: the solution structure of acyl carrier protein. FEBS Lett. 242 (1989), pp. 218-224.

16. G. Cornilescu, F. Delaglio and A. Bax, Protein backbone angle restraints from searching a database for chemical shift and sequence homology. J. Biomol. NMR 13 (1999), pp. 289-302.

17. D. Wishart and B. Sykes, The ${ }^{13} \mathrm{C}$ chemical-shift index: a simple method for the identification of protein secondary structure using ${ }^{13} \mathrm{C}$ chemical-shift data. J Biomol. NMR 4 (1994), pp. 171-180.

18. R.A. Laskowski, M.W. MacArthur, D.S. Moss and J.M. Thornton, Procheck: a program to check the stereochemical quality of protein structures. J. Appl. Crystrallogr. 26 (1993), pp. 283-291.

19. R. Laskowski, J. Rullmannn, M. MacArthur, R. Kaptein and J.M. Thornton, Aqua and procheck-nmr: programs for checking the quality of protein structures solved by Nmr. Biomol. NMR 8 (1996), pp. 477-486.

20. C.O. Rock and J.E. Cronan, Jr., Re-evaluation of the solution structure of acyl carrier protein. J. Biol. Chem. 254 (1979), pp. 9778-9785.
21. C.O. Rock, J.E. Cronan, Jr. and I.M. Armitage, Molecular properties of acyl carrier protein derivatives. J. Biol. Chem. 256 (1981), pp. 2669-2674.

22. J.E. Cronan, Jr., Molecular properties of short chain acyl thioesters of acyl carrier protein. J. Biol. Chem. 257 (1982), pp. 50135017.

23. P.J. Jones, E.A. Cioffi and J.H. Prestegard, Fluorine-19\}-proton heteronuclear nuclear Overhauser effect studies of the acyl chain-binding site of acyl carrier protein. J. Biol. Chem. 262 (1987), pp. 8963-8965.

24. K.H. Mayo and J.H. Prestegard, Acyl carrier protein from E. coli. Structural characterization of short-chain acylated acyl carrier proteins by NMR. Biochemistry 24 (1985), pp. 7834-7838.

25. K. Parris, Crystal structures of substrate binding to Bacillus subtilis olo-(acyl carrier protein) synthase reveal a novel trimeric arangement of molecules resulting in three active sites. Structure 8 (2000), pp. 883-895.

26. M.C. Oswood, Y. Kim, J.B. Ohlrogge and J.H. Prestegard, Structural homology of spinach acyl carrier protein and Escherichia coli acyl carrier protein based on NMR data. Proteins: Struct. Funct. Genet. 27 (1997), pp. 131-143.

27. R. Ghose, O. Geiger and J.H. Prestegard, NMR investigations of the structural properties of the nodulation protein, NodF, from Rhizobium leguminosarum and its homology with Escherichia coli acyl carrier protein. FEBS Lett. 388 (1996), pp. 66-72.

28. G.M. Clore and A.M. Gronenborn, NMR structure determination of proteins and protein complexes larger than $20 \mathrm{kDa}$. Curr. Opin. Chem. Biol. 2 (1998), pp. 564-570.

29. J. Kuszewski, J. Qin, A.M. Gronenborn and G.M. Clore, The impact of direct refinement against ${ }^{13} \mathrm{C}$.alpha., and ${ }^{13} \mathrm{C}$.beta. chemical shifts on protein structure determination by NMR. J. Magn. Reson. Ser. B 106 (1995), pp. 92-96.

30. J. Kuszewski, A.M. Gronenborn and G.M. Clore, Improving the quality of NMR and crystallographic protein structures by means of a conformational database potential derived from structure databases. Protein Sci. 5 (1996), pp. 1067-1080.

31. G.M. Clore and A.M. Gronenborn, Structures of larger proteins, protein-ligand and protein-DNA complexes by multidimensional heteronuclear NMR. Protein Sci. i (1994), pp. 372-390.

32. T. Weber, R. Baumgartner, C. Renner, M.A. Marahiel and T.A. Holak, Solution structure of PCP, a prototype for the peptidyl carrier domains of modular peptide synthetases. Structure 8 (2000), pp. 407-418.

33. L.E. Kay, Pulsed field gradient multi-dimensional NMR methods for the study of protein structure and dynamics in solution. Prog. Biophys. Molec. Biol 63 (1995), pp. 110-126.

34. S.M. Pascal, D.R. Muhandiram, T. Yamazaki, J.D. Forman-Kay and L.E. Kay, Simultaneous acquisition of ${ }^{15} \mathrm{~N}$ - and ${ }^{13} \mathrm{C}$-edited NOE spectra of proteins dissolved in $\mathrm{H}_{2} \mathrm{O}$. J. Magn. Reson. Ser. B 103 (1994), pp. 197-201.

35. G.Y. Xu et al.T.S. Harvey, Solution structure of a cellulose-binding domain from cellulomonas fimi by nuclear magnetic resonance spectroscopy. Biochemistry 34 (1995), pp. 6993-7009.

36. F. Delaglio, S. Grzesiek, G.W. Vuister, G. Zhu, J. Pfeifer and A. Bax, NMRPipe: A multidimensional spectral processing system based on UNIX pipes. J. Biomol. NMR 6 (1995), pp. 277-293.

37. G. Zhu and A. Bax, Improved linear prediction of damped NMR signals using modified "forward-backward" linear. J. Magn. Reson. 100 (1992), pp. 202-207.

38. D.S. Garrett, R. Powers, A.M. Gronenborn and G.M. Clore, A common sense approach to peak picking in two-, three-, and four-dimensional spectra using automatic computer analysis of contour diagrams. J. Magn. Reson. 95 (1991), pp. 214-220. 
39. D.R. Muhandiram and L.E. Kay, Gradient-enhanced triple-resonance three-dimensional NMR experiments with improved sensitivity. J. Magn. Reson. Ser. B 103 (1994), pp. 203-216.

40. O. Zhang, L.E. Kay, J.P. Olivier and J.D. Forman-Kay, Backbone $1 \mathrm{H}$ and ${ }^{15} \mathrm{~N}$ resonance assignments of the $\mathrm{N}$-terminal $\mathrm{SH} 3$ domain of drk in folded and unfolded states using enhanced-sensitivity pulsed field gradient NMR techniques. J. Biomol. NMR 4 (1994), pp. 845-858.

41. K. Wuthrich, M. Billeter and W. Braun, Pseudo-structures for the 20 common amino acids for use in studies of protein conformation by measurements of intramolecular proton-proton distance constraints with nuclear magnetic resonance. J. Mol. Biol. 169 (1983), pp. 949-961.

42. G.M. Clore, A.M. Gronenborn, M. Nilges and C.A. Ryan, Threedimensional structure of potato carboxypeptidase inhibitor in solution. A study using nuclear magnetic resonance, distance geometry, and restrained molecular dynamics. Biochemistry 26 (1987), pp. 8012-8023.

43. G. Wagner, W. Braun, T.F. Havel, T. Schaumann, N. Go and K. Wuethrich, Protein structures in solution by nuclear magnetic resonance and distance geometry. The polypeptide fold of the basic pancreatic trypsin inhibitor determined using two different algorithms, DISGEO and DISMAN. J. Mol. Biol. 196 (1987), pp. 611-639.
44. M. Nilges, A.M. Gronenborn, A.T. Bruenger and G.M. Clore, Determination of three-dimensional structures of proteins by simulated annealing with interproton distance restraints. Application to crambin, potato carboxypeptidase inhibitor and barley serine proteinase inhibitor 2. Protein Eng. 2 (1988), pp. 27-38.

45. G.M. Clore, E. Appella, M. Yamada, K. Matsushima and A.M. Gronenborn, Three-dimensional structure of interleukin 8 in solution. Biochemistry 29 (1990), pp. 1689-1696.

46. A.T. Brunger. X-PLOR Version 3.1 Manual, Yale University Press, New Haven, CT (1993).

47. J. Kuszewski, A.M. Gronenborn and G.M. Clore, Improvements and extensions in the conformational database potential for the refinement of NMR and x-ray structures of proteins and nucleic acids. J. Magn. Reson. 125 (1997), pp. 171-177.

48. Koradi, R., Billeter, M., and Wuethrich, K. (1996). MOLMOL: a program for display and analysis of macromolecular structures. J. Mol. Graphics 14, 51-55 plates, 29-32.

\section{Accession Numbers}

Atomic coordinates and the NMR chemical shift assignments for the restrained minimized mean structure of ACP have been deposited in the RCSB Protein Data Bank (PDB ID code 1HY8). 